\title{
Astilbin-induced inhibition of the PI3K/AKT signaling pathway decelerates the progression of osteoarthritis
}

\author{
CHUNLIN CHEN $^{1 *}$, MAO YANG $^{1 *}$, YUJIANG CHEN $^{1}$, YAOYAO WANG $^{1}$, \\ KUN WANG $^{1}$, TENGXIAN LI ${ }^{1}$, QING HU ${ }^{1}$, WENJING ZHANG ${ }^{1}$ and JINGFU XIA ${ }^{2}$ \\ ${ }^{1}$ Department of Pathology and ${ }^{2}$ Miao Medicine Department, The First Affiliated Hospital \\ of Guizhou University of Traditional Chinese Medicine, Guiyang, Guizhou 550001, P.R. China
}

Received January 29, 2020; Accepted June 23, 2020

DOI: $10.3892 /$ etm.2020.9048

\begin{abstract}
Degeneration and destruction of articular cartilage are the key characteristics of osteoarthritis (OA). In recent studies, the use of astilbin (AST), the primary active ingredient of Astilbe chinensis, has been shown to correlate with a reduction in inflammatory disease symptoms. The present study aimed to investigate the effects and mechanisms of AST on OA. A rat model of OA was constructed and in vivo experiments were performed using the AST, PBS, OA and control groups. The cartilage tissues of each group were assessed by hematoxylin and eosin and toluidine blue staining. The gene expression levels of interleukin (IL)-1 $\beta$, tumor necrosis factor (TNF)- $\alpha$, IL-6, AKT, PI3K and other related proteins were analyzed by reverse transcription-quantitative PCR and western blot analysis. AST was found to significantly inhibit IL-1 $\beta$ and TNF- $\alpha$ protein expression; this further confirmed that IL-1 $\beta$, TNF- $\alpha$ and PI3K mRNA expression was downregulated, indicating that the protective mechanism of AST is associated with the PI3K/AKT pathway. Overall, the results of the present study demonstrate that AST can improve OA symptoms by downregulating the PI3K/AKT signaling pathway, and may therefore be a potential therapeutic option for patients with OA.
\end{abstract}

\section{Introduction}

Osteoarthritis (OA) is a degenerative joint disease affecting millions globally. OA is one of the primary types of arthritis

Correspondence to: Mr. Jingfu Xia, Miao Medicine Department, The First Affiliated Hospital of Guizhou University of Traditional Chinese Medicine, 71 Baoshan North Road, Guiyang, Guizhou 550001, P.R. China

E-mail: rkd26c@163.com; xiajingfu12@163.com

${ }^{*}$ Contributed equally

Key words: astilbin, osteoarthritis, PI3K/AKT signaling pathway, inflammatory cytokines and is predicted to become the fourth leading cause of disability by 2020 (1). The pathological features of knee osteoarthritis (KOA) result from the chronic degeneration of chondrocytes $(2,3)$. Since individuals $>65$ years of age suffer from different degrees of KOA, its prevalence has steadily increased. KOA-related cartilage destruction, synovitis and osteophyte hyperplasia have become the main causes of disability in adults, especially in the elderly (1). As a progressive degenerative joint disease, the continual degeneration of articular cartilage promotes bone-to-bone friction, resulting in severe pain, stiffness and disability. A close correlation between inflammatory and catabolic changes and the occurrence and development of OA has been reported. However, the pathogenetic mechanisms of OA are yet to be fully elucidated (4). Therefore, current OA treatment options are limited, and there are no treatments that can effectively prevent its progression. With the recent development of biological agents, the TNF- $\alpha$ inhibitor etanercept has become an effective agent that provides biological support for tissue regeneration and repair (5). Total knee arthroplasty can only be used to treat patients with advanced OA, while those with mild disease are largely treated with drugs, including joint nutrients, non-steroidal anti-inflammatories, opioid analgesics and antipyretic analgesics. To a certain extent, patient joint mobility and clinical symptoms can be improved, but these treatments cannot slow or reverse the degeneration process, and the side effects of long-term use are significant (6). Therefore, the identification of safe and efficient treatments for OA is paramount.

At present, increasing numbers of natural plant compounds are being used to treat diseases and their associated complications. Astilbe chinensis is a traditional Chinese medicine which is commonly used to treat rheumatoid and skeletal muscle diseases, of which astilbin (AST) is one of the primary dihydroflavonol glycoside constituents $(7,8)$. Previous studies have shown that AST can significantly decrease the levels of serum interleukin (IL)-1, tumor necrosis factor- $\alpha$ (TNF- $\alpha$ ) and IL- 6 (as well as the activities of their associated protein targets) in rats with rheumatoid arthritis (9).

Previous studies have demonstrated that the NF- $\kappa \mathrm{B}$ inhibitor $\alpha$ and PI3K/AKT signaling pathways serve key roles in cartilage degeneration (10). In addition, TNF- $\alpha$ and IL- 6 may be used as downstream regulators of the 
nuclear PI3K/AKT/NF- $\kappa \mathrm{B}$ signaling, and thus mediate the inflammatory response. Therefore, inhibiting the functions of such inflammatory pathways is an acknowledged option for the treatment of OA (11). Since AST downregulates the expression of TNF- $\alpha$, we hypothesized that it may also serve a preventive role in OA. In the present study, papain-induced rats were used to evaluate the therapeutic effects of AST on $\mathrm{OA}$, and to investigate its potential anti-inflammatory mechanisms.

\section{Materials and methods}

AST and its primary reagents. Pure AST was provided by Shanghai Yuanye Biotechnology Co., Ltd. For experiments, AST was dissolved in phosphate buffered saline (PBS) and diluted with normal saline. Pentobarbital sodium, papain and cysteine were purchased from Guangzhou Chemical General Factory, and hematoxylin, water-soluble eosin Y, toluidine blue $\mathrm{O}$, paraformaldehyde and EDTA were acquired from Takara Bio, Inc. Anti-TNF (cat. no. ab1793), IL-1 (cat. no. ab9722), $\beta$-actin (cat. no. ab8227), AKT (cat. no. ab8805), NF- $\mathrm{B}$ (cat. no. ab28849), PI3K (cat. no. ab140307) and IL-6 (cat. no. ab6672) antibodies were all purchased from Abcam. The quantitative PCR detection and Prime Script RT kits were purchased from Bao Biological Engineering Co. Ltd., and Takara Biotechnology Co. Ltd., respectively.

Animals. A total of 24 male Sprague-Dawley rats (age, 12 weeks; weight, 200-250 g) were purchased from and raised in the Experimental Animal Center of Guiyang College of Traditional Chinese Medicine (Guiyang, China), in line with the specific pathogen-free conditions of the College Animal Care and Use Committee. The rats were allowed free access to food and water.

All experiments conducted in the present study were approved by the Ethics Committee of Guiyang College of Traditional Chinese Medicine.

Animal model establishment and intervention. The OA model was constructed using 12-week-old rats that were randomly divided into the following 4 groups ( $n=6$ per group): i) AST; ii) PBS; iii) OA; and iv) control. AST was dissolved in PBS, thus the solvent was used as a control to eliminate interference factors. Rats in the AST, PBS and OA groups were injected with a mixed solution $(0.25 \mathrm{ml} / \mathrm{kg})$ of $4 \%(\mathrm{w} / \mathrm{w})$ papain and $0.3 \mathrm{~mol} / 1$ cysteine at the knee joint on days 1,3 and 5 . The control group did not receive surgical intervention. After successful establishment of the OA model, $3 \mathrm{mg} / \mathrm{kg}$ AST was administered to the AST group by gavage, and the PBS group received an equal volume of PBS only; the other two groups were treated with normal saline once a day for 4 weeks. At 4 weeks post-surgery, the rats were euthanized with pentobarbital sodium, and the knee joint tissues were collected for further analysis.

Hematoxylin and eosin $(H \& E)$ staining. Cartilage tissues were collected from the knee joints of each rat, fixed in $4 \%$ paraformaldehyde for $24 \mathrm{~h}$, and then dehydrated using a graded alcohol series. The tissue were embedded in paraffin and sliced into sections of $4 \mu \mathrm{m}$ thickness. After routine dewaxing, the sections were stained with hematoxylin for $8 \mathrm{~min}$, washed with tap water for $1 \mathrm{~min}$, and stained for $3 \mathrm{~min}$ in eosin solution. The sections were then sequentially sealed by graded alcohol dehydration, transparent xylene and neutral gum.

Toluidine blue staining. After routine dewaxing, the tissue sections were stained with $0.5 \%$ toluidine blue solution for $30 \mathrm{~min}$ and washed with tap water. After separation for $5 \mathrm{sec}$ in $0.5 \%$ glacial acetic acid solution, the sections were washed with distilled water and subsequently sealed by gradient alcohol dehydration, transparent xylene and neutral gum (as aforementioned). The average optical density of toluidine blue was then analyzed.

Western blot analysis. The articular cartilage was placed in a mortar, liquid nitrogen was added, and the samples were immediately ground. After grinding, the proteins were extracted using $1 \mathrm{~g} / 4 \mathrm{ml}$ RIPA protein lysis buffer at $4^{\circ} \mathrm{C}$. After $2 \mathrm{~h}$ with vibration at $30 \mathrm{~min}$ intervals, the lysates were centrifuged at $219,128 \times \mathrm{g}$ at $4^{\circ} \mathrm{C}$ for $30 \mathrm{~min}$ and quantified using a BCA assay. The supernatants (200 $\mu \mathrm{l}$ each) were transferred to a fresh centrifuge tube and $50 \mu \mathrm{l}$ protein loading buffer was added before boiling for $5 \mathrm{~min}$. Equal amounts of protein were separated using 10\% SDS-PAGE gels, and then transferred onto PVDF membranes for $2 \mathrm{~h}$. After transfer, the membranes were blocked with $5 \%$ skimmed milk at room temperature for $2 \mathrm{~h}$, and then $\beta$-catenin antibody, AKT $(1: 1,000)$, PI3K (1:1,000), TNF- $\alpha(1: 1,500)$, IL-6 (1:1,000) and IL-1 $\beta(1: 1,000)$ primary antibodies were added for overnight incubation at $4^{\circ} \mathrm{C}$. The membranes were washed 3 times with TBST buffer (10 min each), $4 \mathrm{ml}$ secondary goat anti-rabbit IgG/HRP antibody (1:10) was added, and the membranes were incubated at room temperature for $1 \mathrm{~h}$. The membranes were then washed as aforementioned prior to exposure and image development. The grayscale value was analyzed using Image Lab software (Bio-Rad Laboratories, Inc.) and the relative protein expression levels were calculated. The relative expression of $\beta$-actin = actual protein gray value/internal reference gray value.

Reverse transcription-quantitative $(R T-q) P C R$. Total RNA was extracted from the rat cartilage tissues using TRIzol ${ }^{\circledR}$ reagent. According to the manufacturer's instructions, mRNA was reverse transcribed using the PrimeScript RT kit (Takara Bio, Inc.) and qPCR was conducted using the Thermal Cycler Dice $^{\mathrm{TM}}$ real-time system (Takara Bio, Inc.) with $\mathrm{SYBR}^{\circledR}$ Green I dye. Sequence-specific primers were designed to produce products with lengths between 120 and 436 bp (listed in Table I). The average $\mathrm{Ct}$ values were standardized to that of $\beta$-actin, and the results were quantified using the $2^{-\Delta \Delta \mathrm{Cq}}$ method. All experiments were repeated three times.

Statistical analysis. The data were obtained from $\geq 3$ independent experiments and are expressed as the mean \pm standard deviation. Single factor analysis of variance followed by the LSD test was used for comparisons between groups. Data analysis was performed using the SPSS 20.0 statistical software package (IBM Corp.) and $\mathrm{P}<0.05$ was considered to indicate a statistically significant difference. 
Table I. Primer sequences.

\begin{tabular}{lll}
\hline Genes & \multicolumn{1}{c}{ Forward } & Reverse \\
\hline TNF & CTCACCACAAAGGAGAAGCCT & GGTAAGGGAAAGAGGTCGGC \\
IL-6 & CTGATGCTGCCTATTGCCCA & TGCTCAGACTCTCCCTTCTGA \\
IL-1 $\beta$ & CCTTGTCGAGAATGGGCAGT & CAGGGAGGGAAACACACGTT \\
AKT & CACTCCCGGTGAACTCTGAC & CTAAAGGCCGCCCTACACAA \\
PI3K & ACCTGGGAGTGGAGAAACAGA & GTGGGCCACATCACTTAGACA \\
$\beta$-actin & GTGTGGTCAGCCCTGTAGTT & CCTAGAAGCATTTGCGGTGC
\end{tabular}

TNF, tumor necrosis factor; IL, interleukin.
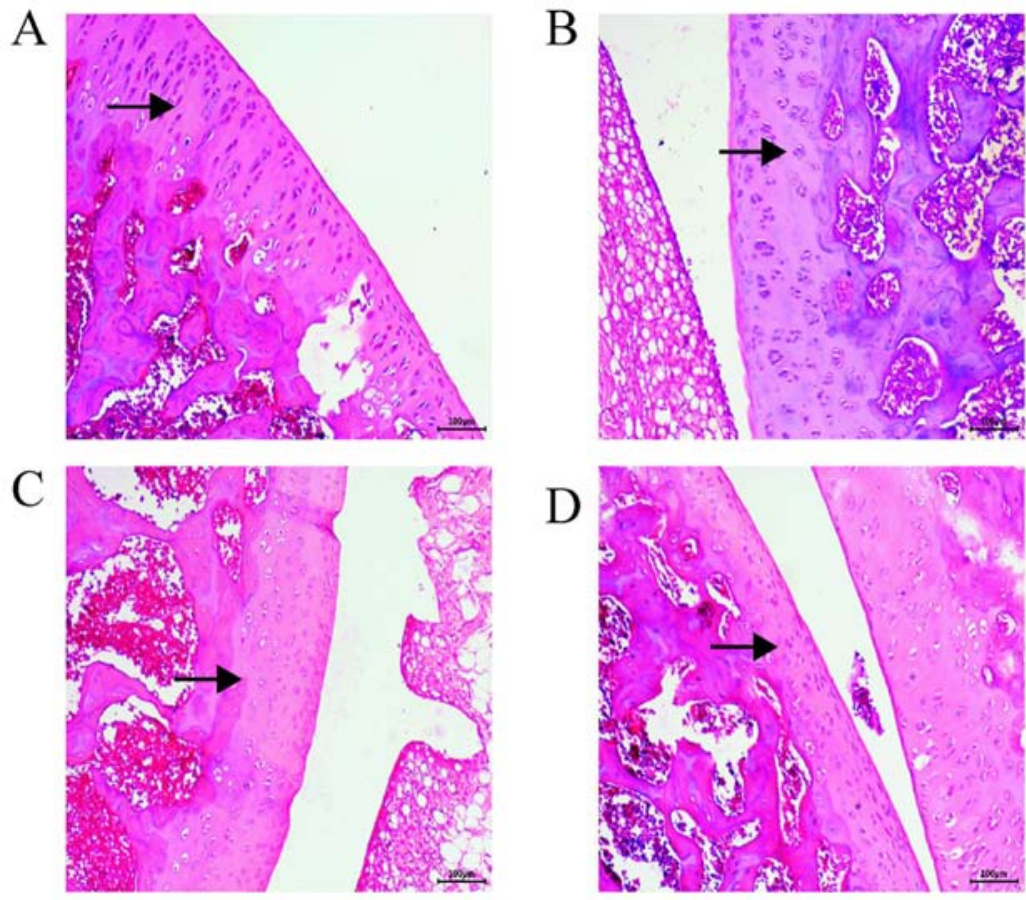

Figure 1. Hematoxylin and eosin staining map of rat cartilage tissue sections. Cartilage matrix in the OA and the PBS groups was lightly stained; arrows indicate chondrocytes, and the cartilage matrix. In the OA and PBS groups, the cartilage was severely damaged, the cartilage layer and cell numbers were significantly reduced, and proteoglycan was lost. In the AST group, the cytoplasm and cartilage matrix were pink and evenly stained, and the chondrocytes were neatly arranged. Surface layers of the control group tissues were smoother than those of the AST group; the cartilage layer was clearly discernible and the tidal line was largely complete. Magnification, x100. (A) AST, (B) PBS, (C) OA and (D) control groups. OA, osteoarthritis; AST, astilbin.

\section{Results}

$H \& E$ staining analysis. H\&E staining revealed that the surface layers of the control group tissues were intact and smoother than those of the AST group, where the cartilage layer was clearly discernible and the tidal line was intact (Fig. 1D). In the PBS group, the articular cartilage was damaged with a rough surface; the cartilage layer was narrower, and the chondrocytes were exposed with a disordered arrangement (Fig. 1B). In the OA group, severe destruction of the cartilage, an obvious decrease in cell numbers, a decrease in the cartilage layer and proteoglycan loss were observed (Fig. 1C). In the AST group, the cartilage surface was smooth, and the structure was normal. The cytoplasm and cartilage matrix were pink and evenly stained, the chondrocytes were neatly arranged and the tide line was intact (Fig. 1A). The AST group exhibited decelerated cartilage destruction and the lowest degree of joint damage, which was similar to the results of the control group. The chondrocytes were more neatly arranged than those in the PBS and OA groups, with decreased levels of cytoclasis.

Toluidine blue staining. Toluidine blue staining revealed that in the control group, the superficial layer of the cartilage was deeply stained with a blueish-purple color; the stained area was large with a uniform color distribution (Fig. 2D). In the PBS group, the staining depth and area were decreased, and large unstained areas were present (Fig. 2B). Tissues in the OA group also possessed large unstained areas, a light blue color, and a significantly lower proteoglycan content than those of the other groups (Fig. 2C). Compared with the OA and PBS groups, the AST group exhibited relatively uniform blueish-purple staining of a higher color depth (Fig. 2A). 

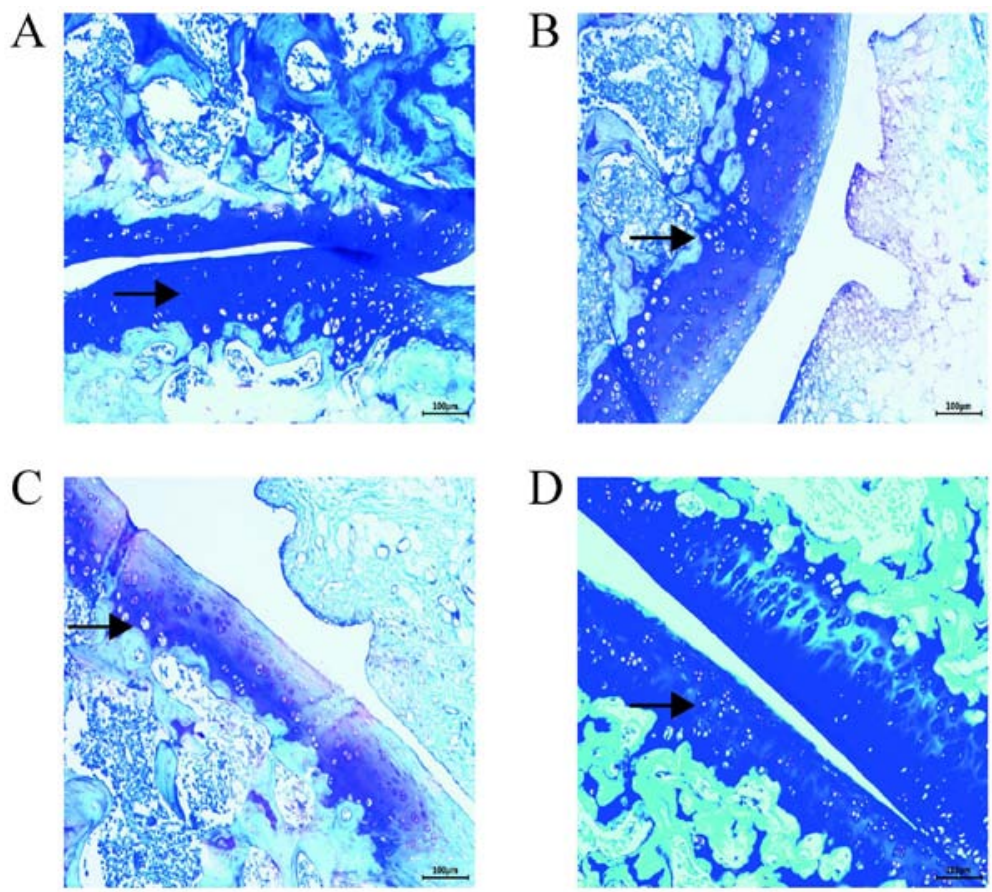

Figure 2. Toluidine blue staining map of rat cartilage tissue sections. Cartilage tissues from the OA and PBS groups were lightly stained; arrows indicate chondrocytes. A deeper staining concentration indicates a greater ability to secrete proteoglycans and decreased cartilage destruction. Magnification, $\mathrm{x} 100$. (A) AST, (B) PBS, (C) OA and (D) control groups. OA, osteoarthritis; AST, astilbin.
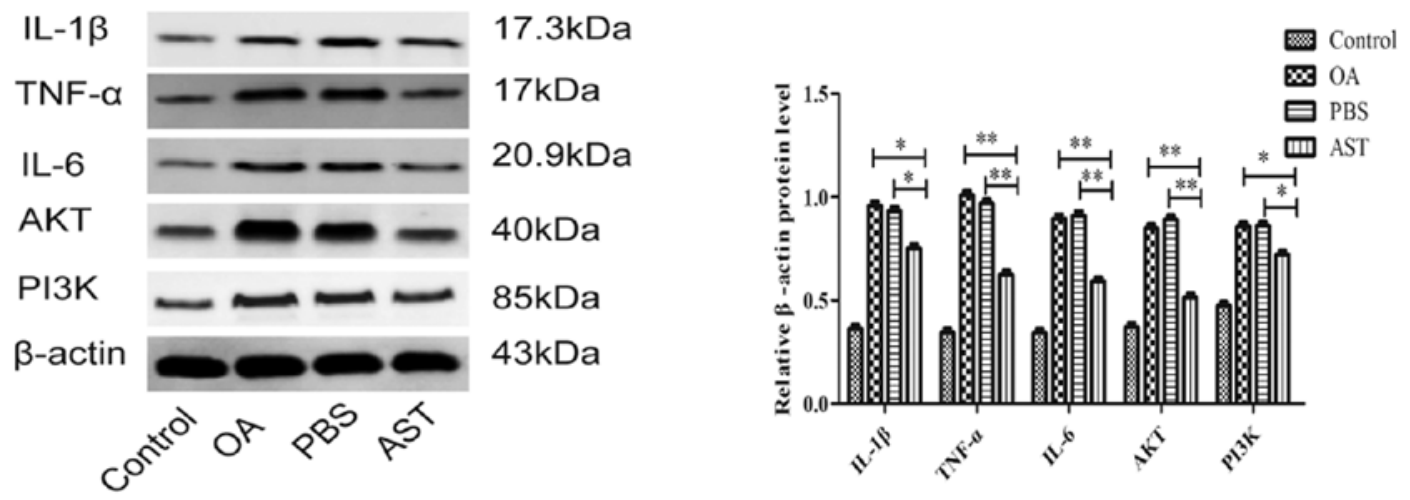

Figure 3. Western blot analysis of IL-1 $\beta$, TNF- $\alpha$, IL-6, AKT and PI3K protein expression in rat cartilage tissue sections. ${ }^{*} \mathrm{P}<0.05$ and ${ }^{* *} \mathrm{P}<0.001$ vs. the PBS and OA groups. OA, osteoarthritis; AST, astilbin; IL, interleukin; TNF- $\alpha$, tumor necrosis factor- $\alpha$.

Western blot analysis. Western blot detection demonstrated that TNF- $\alpha$, IL- 6 and AKT protein expression was significantly lower in the cartilage of the AST group rats than in those of the PBS and OA groups $(\mathrm{P}<0.001)$. The levels of IL-1 $\beta$ and PI3K in the AST group were lower than those in the PBS and OA groups, though the difference was still significant $(\mathrm{P}<0.05)$. Compared with the control group, the relative expression level of each protein was higher in the PBS and OA groups (Fig. 3).

$R T-q P C R$. The results of $\mathrm{RT}-\mathrm{qPCR}$ indicate that when compared with the PBS and OA groups, the mRNA levels of TNF- $\alpha$, IL-6 and AKT were significantly lower in the cartilage of rats in the AST group. Relative to the OA group, the PI3K levels in the AST group tissues were also significantly lower $(\mathrm{P}<0.05)$. There were no significant differences in IL-1 $\beta$ expression between the groups, and the control group displayed the lowest expression levels of all five genes (Fig. 4).

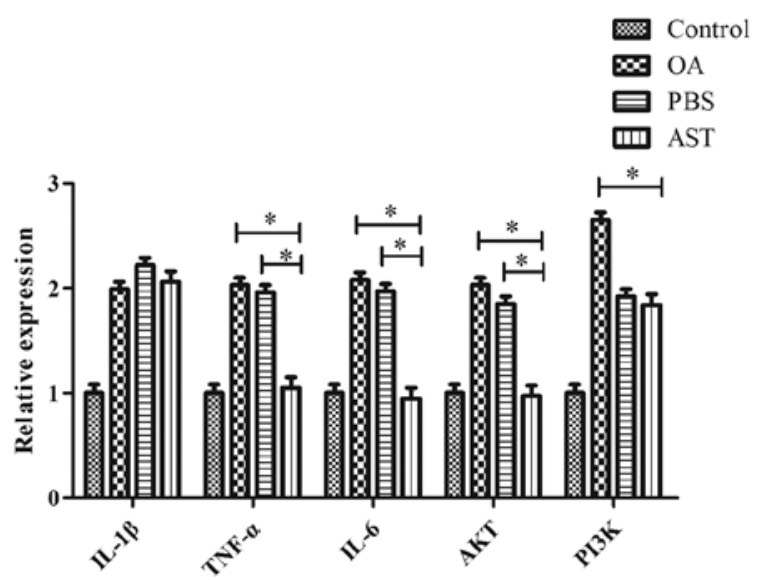

Figure 4. mRNA expression levels of IL- $\beta$, TNF- $\alpha$, IL- 6 , AKT and PI3K in rat cartilage tissue sections. " $\mathrm{P}<0.05$ vs. the $\mathrm{PBS}$ and $\mathrm{OA}$ groups. OA, osteoarthritis; AST, astilbin; IL, interleukin; TNF- $\alpha$, tumor necrosis factor- $\alpha$. 


\section{Discussion}

$\mathrm{OA}$ is the most common joint disease and the primary cause of joint-associated disability worldwide. As the population ages, the prevalence of KOA is expected to continue to rise, and to place a heavy burden on the social health care system (12). Although a variety of risk factors have been associated with the onset of OA in recent decades, the therapeutic effects of physiotherapy remain limited. As such, non-steroidal antiinflammatory drugs are the primary treatment options for OA (13); however, these compounds only temporarily ameliorate the clinical symptoms, rather than slow the development of OA. Therefore, there is an urgent need for drugs that specifically target the molecular components of OA-associated cartilage degradation.

Research into the efficacy and mechanisms of Chinese herbal medicines for the treatment of OA are critical. Previously, Astilbe chinensis was mainly used to treat rheumatism and relieve swelling and pain. Astilbe chinensis contains a series of bioactive components, including flavonoids, mannose-binding lectins and terpenes. In the clinic, it is often used to treat nephritis, rheumatoid arthritis, leptospirosis, bacillary dysentery, urethral infections and various other diseases $(11,14)$. Studies have shown that the rhizome extract of Astilbe chinensis can inhibit the activity of specific inflammatory cells via immunomodulation. However, its mechanism is yet to be elucidated. AST is a dihydroflavonol derivative isolated from the rhizome of Astilbe chinensis, which exerts a variety of pharmacological effects, including antioxidant, antiinflammatory, anti-diabetic and nephrotic properties $(15,16)$. Evidence suggests that AST can reduce collagen-induced arthritis-associated dysfunction by selectively inhibiting lymphocyte function (17) and decreasing the production of matrix metalloproteinases (MMPs) and nitric oxide. Moreover, AST attenuates contact allergies by stimulating IL-10 in lymphocytes (18), and alleviates disease progression in rats prone to systemic lupus erythematosus by inhibiting the functionally activated T and B cells (19). Although AST has been reported to inhibit inflammatory factors and suppress the immune response, there are no reports addressing the application of AST in OA. In addition, the anti-inflammatory mechanisms of AST in the treatment of OA remain to be elucidated. To the best of our knowledge, the present study is the first to report the use of AST in the treatment of OA.

The release of TNF- $\alpha$ and IL- 6 serves a key role in the synovial fluid and serum of patients with OA (20). IL-6 is the primary mediator of cartilage and bone degeneration, the accumulation of inflammatory cells, the persistence of inflammation, and the emergence of rheumatoid factors (21). IL-8 functions to promote the infiltration of immune cells and the recruitment of white blood cells (22). In patients with OA, TNF- $\alpha$ is largely produced by activated macrophages and is believed to be a major contributor to inflammation and joint destruction (23). The results of the present study indicate that AST possesses potential anti-inflammatory properties and can decrease OA-associated cartilage damage. The PI3K/AKT pathway is associated with the pathogenesis of OA, as well as changes in the extracellular matrix (24). Activation of the PI3K/AKT pathway can induce the phosphorylation of $\mathrm{I} \kappa \mathrm{B} \alpha$ and $\mathrm{p} 65$, activating the downstream NF- $\kappa \mathrm{B}$ pathway in chondrocytes, thus increasing the production of MMP and cyclooxygenase-2 (25). Hence, inhibition of the PI3K/AKT pathway has been considered as an option for the treatment of OA.

In the present study, H\&E staining indicated that AST may protect the articular surface and reduce OA-associated damage. According to western blot analysis, AST was found to inhibit the phosphorylation of PI3K and AKT (25). At the same time, the mRNA expression levels of TNF- $\alpha$, IL- 6 , PI3K and AKT were downregulated in the cartilage tissue of rats in the AST group, suggesting that the inflammatory response was retarded, and that inhibiting the inflammatory pathways may provide a favorable environment for articular cartilage repair.

The results of the present study indicate that AST significantly inhibits the expression of inflammation-associated factors by targeting the PI3K/AKT pathway, which enables it to play a significant role in the treatment of OA. AST is believed to regulate and prevent OA by downregulating IL-1 $\beta$ and TNF- $\alpha$ expression, which delays cartilage degeneration and promotes cartilage repair. The present study provides experimental evidence and a theoretical basis, expounding the mechanisms of this traditional Chinese medicine in the treatment of OA. However, there are limitations to the current study. It is necessary to further investigate the potential synergism between NF- $\mathrm{KB}$ and other related factors (including Toll-like receptors and mitogen-activated protein kinases), and the relevant molecular pathways associated with their inflammatory mechanisms. This will be the focus of subsequent studies.

\section{Acknowledgements}

Not applicable.

\section{Funding}

The study was supported by the Science and Technology Cooperation Program of the Department of Science and Technology of Guizhou Province and the First Affiliated Hospital of Guizhou University of Traditional Chinese Medicine (fund nos. LH[2016] and 7508).

\section{Availability of data and materials}

The datasets used and/or analyzed during the current study are available from the corresponding author on reasonable request.

\section{Authors' contributions}

CC and MY conceived the study and wrote the manuscript. YC established the animal model. YW and KW were responsible for $\mathrm{H} \& \mathrm{E}$ staining and Toluidine blue staining. TL and $\mathrm{QH}$ were responsible for western blot analysis. WZ and JX performed real-time fluorescence quantitative PCR. All authors read and approved the final manuscript.

\section{Ethics approval and consent to participate}

The study was approved by the Ethics Committee of the Guiyang College of Traditional Chinese Medicine (Guiyang, China). 


\section{Patient consent for publication}

Not applicable.

\section{Competing interests}

The authors declare that they have no competing interests.

\section{References}

1. Thielen NGM, van der Kraan PM and van Caam APM:TGF/BMP signaling pathway in cartilage homeostasis. Cells 8: E969, 2019.

2. Neogi T: Structural correlates of pain in osteoarthritis. Clin Exp Rheumatol 35 (Suppl 107): 75-78, 2017.

3. Zhao Y, Li Z, Wang W, Zhang H, Chen J, Su P, Liu L and Li W: Naringin protects against cartilage destruction in osteoarthritis through repression of NF- $\kappa \mathrm{B}$ signaling pathway. Inflammation 39 : 385-392, 2016

4. Ahn H, La JH, Chung JM, Miao H, Zhong C, Kim M, An K, Lyon D, Choi E and Fillingim RB: The relationship between $\beta$-endorphin and experimental pain sensitivity in older adults with knee osteoarthritis. Biol Res Nurs 21: 400-406, 2019.

5. Mehana EE, Khafaga AF and El-Blehi SS: The role of matrix metalloproteinases in osteoarthritis pathogenesis: An updated review. Life Sci 234: 116786, 2019.

6. Fei J, Liang B, Jiang C, Ni H and Wang L: Luteolin inhibits IL-1 $\beta$-induced inflammation in rat chondrocytes and attenuates osteoarthritis progression in a rat model. Biomed Pharmacother 109: 1586-1592, 2019.

7. He X, Yi T, Tang Y, Xu J, Zhang J, Zhang Y, Dong L and Chen H: Assessing the quality of Smilacis Glabrae Rhizoma (Tufuling) by colormetrics and UPLC-Q-TOF-MS. Chin Med 11: 33, 2016.

8. Hao G, Zheng J, Huo R, Li J, Wen K, Zhang Y and Liang G: Smilax glabra Roxb targets $\mathrm{Akt}^{\mathrm{p}-\mathrm{Thr} 308}$ and inhibits Akt-mediated signaling pathways in SGC7901 cells. J Drug Target 24: 557-565, 2016.

9. Dong L, Zhu J, Du H, Nong H, He X and Chen X: Astilbin from Smilax glabra Roxb. Attenuates inflammatory responses in complete Freund's adjuvant-induced arthritis rats. Evid Based Complement Alternat Med 2017: 8246420, 2017.

10. Huang X, Xi Y, Mao Z, Chu X, Zhang R, Ma X, Ni B, Cheng H and You H: Vanillic acid attenuates cartilage degeneration by regulating the MAPK and PI3K/AKT/NF-KB pathways. Eur J Pharmacol 859: 172481, 2019.

11. Lu CL, Zhu W, Wang M, Xu XJ and Lu CJ: Antioxidant and antiinflammatory activities of phenolic-enriched extracts of Smilax glabra. Evid Based Complement Alternat Med 2014: 910438, 2014.
12. Bijlsma JW, Berenbaum F and Lafeber FP: Osteoarthritis: An update with relevance for clinical practice. Lancet 377 : 2115-2126, 2011.

13. Nguyen PT and Marquis RE: Antimicrobial actions of $\alpha$-mangostin against oral streptococci. Can J Microbiol 57: 217-225, 2011.

14. Wang M, Yang XB, Zhao JW, Lu CJ and Zhu W: Structural characterization and macrophage immunomodulatory activity of a novel polysaccharide from Smilax glabra Roxb. Carbohydr Polym 156: 390-402, 2017.

15. Lu CL, Zhu YF, Hu MM, Wang DM, Xu XJ, Lu CJ and Zhu W: Optimization of astilbin extraction from the rhizome of Smilax glabra, and evaluation of its anti-inflammatory effect and probable underlying mechanism in lipopolysaccharide-induced RAW264.7 macrophages. Molecules 20: 625-644, 2015.

16. Yan R and Xu Q: Astilbin selectively facilitates the apoptosis of interleukin-2-dependent phytohemagglutinin-activated Jurkat cells. Pharmacol Res 44: 135-139, 2001.

17. Cai Y, Chen T and Xu Q: Astilbin suppresses collagen-induced arthritis via the dysfunction of lymphocytes. Inflamm Res 52: 334-340, 2003

18. Fei M, Wu X and Xu Q: Astilbin inhibits contact hypersensitivity through negative cytokine regulation distinct from cyclosporin A. J Allergy Clin Immunol 116: 1350-1356, 2005.

19. Guo L, Liu W, Lu T, Guo W, Gao J, Luo Q, Wu X, Sun Y, Wu X, Shen Y, et al: Decrease of functional activated T and B cells and treatment of glomerulonephitis in lupus-prone mice using a natural flavonoid astilbin. PLoS One 10: e0124002, 2015.

20. Kaneko S, Satoh T, Chiba J, Ju C, Inoue K and Kagawa J: Interleukin-6 and interleukin-8 levels in serum and synovial fluid of patients with osteoarthritis. Cytokines Cell Mol Ther 6: 71-79, 2000.

21. Szekanecz Z and Koch AE: Successes and failures of chemokinepathway targeting in rheumatoid arthritis. Nat Rev Rheumatol 12: 5-13, 2016.

22. Guha M and Mackman N: LPS induction of gene expression in human monocytes. Cell Signal 13: 85-94, 2001

23. Mateen S,Zafar A, Moin S, Khan AQ and Zubair S: Understanding the role of cytokines in the pathogenesis of rheumatoid arthritis. Clin Chim Acta 455: 161-171, 2016.

24. Chen J, Crawford R and Xiao Y: Vertical inhibition of the PI3K/ Akt/mTOR pathway for the treatment of osteoarthritis. J Cell Biochem 114: 245-249, 2013.

25. Yuan FL, Xu RS, Jiang DL, He XL, Su Q, Jin C and Li X: Leonurine hydrochloride inhibits osteoclastogenesis and prevents osteoporosis associated with estrogen deficiency by inhibiting the NF- $\kappa \mathrm{B}$ and PI3K/Akt signaling pathways. Bone 75: 128-137, 2015.

This work is licensed under a Creative Commons Attribution-NonCommercial-NoDerivatives 4.0 International (CC BY-NC-ND 4.0) License. 